\title{
On the Leverage of Superimposed Training for Energy-Efficient Spectrum Sensing in Cognitive Radio
}

\author{
Lizeth Lopez-Lopez \\ Faculty of Sciences \\ Autonomous University of San Luis Potosi (UASLP) \\ San Luis Potosi, Mexico \\ lizeth.lopez.lpz@alumnos.uaslp.edu.mx
}

Sami A. Aldalahmeh

Faculty of Engineering and Technology

Al-Zaytoonah University of Jordan

Amman, Jordan

s.aldalahmeh@ieee.org

\author{
Marco Cardenas-Juarez \\ Faculty of Sciences \\ UASLP \\ San Luis Potosi, Mexico \\ marco.cardenas@uaslp.mx
}

\author{
Enrique Stevens-Navarro \\ Faculty of Sciences \\ UASLP \\ San Luis Potosi, Mexico \\ estevens@galia.fc.uaslp.mx
}

\author{
Marcos Katz \\ Centre for Wireless Communications \\ University Of Oulu \\ Oulu, Finland \\ Marcos.Katz@oulu.fi
}

\begin{abstract}
The efficient utilization of the radio-electric spectrum (or simply spectrum) is essential to satisfy the everincreasing amount of bandwidth required by future wireless communications networks. Cognitive radio (CR) networks aim to improve this efficiency by dynamically exploiting the underutilized spectrum (also called spectrum opportunities). To identify these transmission opportunities, cognitive users might draw on spectrum sensing, although this task increases the energy consumption. For battery-powered terminals, this increment might represent a challenge, also considering that spectrum sensing must be recurrently performed. For a scenario in which the $C R$ user first senses the spectrum and then, if allowed, transmit data, the average energy consumption depends on the time used for spectrum sensing and for data transmission, which also impacts the spectrum-efficiency. Thus, improving the energy-efficiency might implicate a reduction on the spectrum-efficiency. This paper analyses the energy-efficiency in the context of spectrum sensing of superimposed training-based transmissions, showing the advantages of using an enhanced spectrum sensing method in terms of the relationship between the spectrum and energyefficiency.
\end{abstract}

Index Terms-spectrum sensing, energy-efficiency, cognitive radio, superimposed training

\section{INTRODUCTION}

The increasing emergence of new wireless communications services and applications imposes more challenges to future wireless communication networks, such as $6 \mathrm{G}$ networks. In this sense, it is a fact that more radio-bandwidth will be needed to cope with the requirements of the growing quantity of wirelessly connected users and devices. Although higher frequency bands might be used in future for certain scenarios [1], the spectrum shortage faced today challenges the availability of radio-spectrum resources. Recent spectral occupation studies have shown, beyond the inefficiency of fixed spectrum allocation policies and the current waste of this vital resource, the possibility of obtaining more radio-bandwidth from the underutilized spectrum. Cognitive radio (CR) is a technology that has been proposed to allow the utilization of the spectrum in a more efficient way, which in consequence diminishes the mentioned waste of this resource. In a CR network, two types of users are defined: i) primary users (PUs), which own a spectrum license to use a portion of the spectrum; and ii) secondary users (SUs) or cognitive users, which can opportunistically access a determined PU's frequency band, i.e., when the PU is not transmitting data. In this context, SUs must first know whether PUs are transmitting data or not. Such information can be obtained by means of spectrum sensing, although this task might represent some challenges for SUs [2]. On the one hand, detecting the PU's signal under very low signal-to-noise ratio (SNR) schemes might be very though, which might cause interference to PUs' transmissions or loss of opportunities for SUs' transmissions, degrading the performance of both PUs and SUs networks [3]. On the other hand, spectrum sensing is a task that is recurrently performed, increasing the energy consumption of the SUs' terminals. For battery-powered terminals, any increment on the energy consumption might represent a challenge [4]. Many factors influence the energy-efficiency of a CR system. For example, the total frame duration and its structure, i.e., how the frame is divided between the sensing phase and the transmission phase. For periodic spectrum sensing and a given total frame duration, if the time used for spectrum sensing is too long, the energy consumed in this phase increases and, even though the accuracy on detecting the PU might be improved, the time left for data transmission might be too short. Thus, it represents a waste in energy and the SU's throughput is reduced. On the other hand, if the time used for sensing is too short, the detection performance is affected, which might 


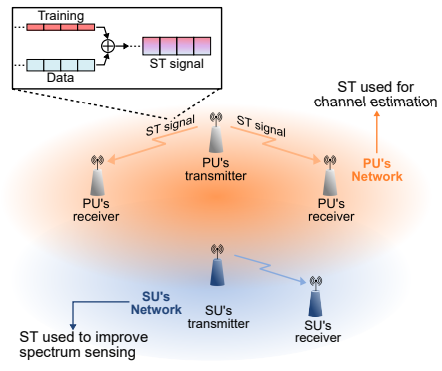

Fig. 1. Illustration of the ST implementation at the PU's transmitter and its context in each network.

result in loss of opportunities for data transmission or in interference to the PU's transmissions, thus decreasing the SU's throughput and wasting energy [4]. Hence, the sensing duration and the detection performance, determined by the spectrum sensing algorithm, affects the spectrum-efficiency (in terms of the throughput measured in bits/s/Hz) and the energy-efficiency (measured in bits/Joule/Hz). Thus, there is a tradeoff between these two efficiency metrics and improving one might result in a decrease of the other one [5]. Studies about improving the energy-efficiency have been carried out using the energy detection algorithm, where the objective has been to find the sensing parameters that improves the energy-efficiency under the condition of sufficient protection to PU's transmissions, e.g., the sensing and transmission duration [4], and decision threshold in a cooperative spectrum sensing scenario [5]. In this paper, the energy-efficiency is studied and its relationship with the spectrum-efficiency. Motivated by the advantages of using an enhanced method for spectrum sensing to improve the detection performance and to show how this impacts the aforementioned tradeoff, the analysis is carried out using the simplified version of the superimposed training combined approach for a reduce phase (SCAR-Phase) of spectrum sensing. This method takes advantage of the superimposed training (ST) information in the PU's signal, if included. Moreover, the SCAR-Phase sensing allows SUs to synchronize with the transmitted training sequence to exploit it during the spectrum sensing [6]. Thus, in addition to the advantages that the usage of ST implies in the PU's network and the SU's network, as shown in Fig. 1, this technique might benefit also the energy-efficiency of SUs terminals.

The rest of the paper is organized as follows: In Section 2 , the spectrum sensing model is described along with the detection performance of the sensing method used in the analysis. Section 3 describes how the energy-efficiency and the spectrum-efficiency are obtained under the considered CR scenario. The results are analyzed in Section 4 and finally, Section 5 concludes the work.

\section{Spectrum Sensing Model}

Assume a CR scenario with a PU and a SU, where the former employs ST for channel estimation in its own network and the latter is interested in using the frequency band of the $\mathrm{PU}$ in an opportunistic way. In this regard, assume that the SU

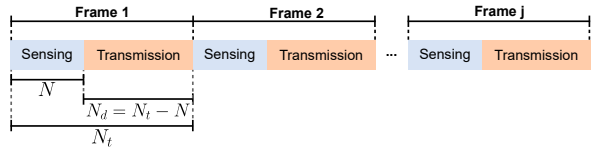

Fig. 2. Frame structure of periodic spectrum sensing.

periodically performs the spectrum sensing task in accordance to the frame structure shown in Fig. 2. Thus, considering a constant frame duration $N_{t}$ (number of samples), the SU collects $N$ samples and uses them to decide whether the PU is transmitting data or not. In the latter case, the SU transmits data for the duration of $N_{d}=N_{t}-N$ samples. Otherwise, it waits until the next sensing phase. When deciding the presence or absence of the PU's signal two errors can be made: i) deciding the presence of the PU when it is absent (i.e. a false alarm), or ii) deciding the absence of the PU when it is present (i.e. a missed detection). The frequency at which these two errors occur (i.e. the probability of false alarm and the probability of missed detection) determines the detection performance of spectrum sensing task and consequently, the performance of the SU's network. Hence, the spectrum sensing algorithm or method must exhibit a low probability of false alarm, to exploit the greater number of spectrum opportunities, and a low probability of missed detection, to reduce the interferences to PU's transmissions. In order to improve the detection performance, assume that the SU knows the PU's training information and takes advantage of it by employing the simplified version of the SCAR-Phase sensing, proposed in [6], which also allows the SU to synchronize with the training sequence transmitted by the PU. This sensing method consists of an energy detection part and a ST-based metric that exploits the training information included in the PU's transmitted signal, as shown in Fig. 3, where the synchronization block can be implemented using the method proposed in [7], $\hat{\tau}$ in the diagram denotes the estimate of $\tau$, explained later, and $*$ denotes complex conjugate.

\section{A. Simplified SCAR-Phase Sensing Detection Performance}

The detection performance of the simplified SCAR-Phase sensing in [6] is characterized by the probability of false alarm and the probability of detection (i.e., the probability of correctly decides the presence of the PU). Assuming that each sample $x[n]$ (with $n$ denoting the sample index) of the received signal $\mathbf{x}=[x[0], x[1], \ldots x[N-1]]^{T}$ (with the superscript $T$ denoting transpose) has the following distribution:

$$
x[n] \sim \begin{cases}\mathcal{C N}\left(0, \sigma^{2}\right) & \text { under } \mathcal{H}_{0}, \\ \mathcal{C N}\left(\theta e^{j \phi} t[n-\tau], \theta^{2} \sigma_{d}^{2}+\sigma^{2}\right) & \text { under } \mathcal{H}_{1},\end{cases}
$$

where $\mathcal{H}_{0}$ and $\mathcal{H}_{1}$ represents the null hypothesis (i.e., when the PU is absent) and alternative hypothesis (i.e., when the $\mathrm{PU}$ is present), respectively. Moreover, $\sigma^{2}$ denotes the noise variance, $\sigma_{d}^{2}$ represents the power of the PU's data signal, and $t[n-\tau]$ is the known training signal with an unknown shift in time given by $\tau$, which represents the lack of training sequence synchronization [7]. Furthermore, $\theta$ and $\phi$ denotes the 


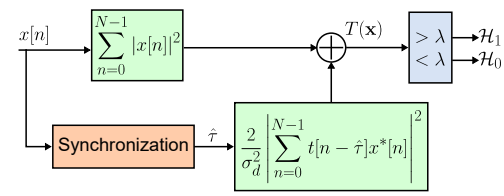

Fig. 3. Block diagram of the simplified SCAR-Phase sensing.

amplitude and phase of the wireless channel gain, respectively. Then, the probability of false alarm is given by

$$
\begin{aligned}
P_{\mathrm{FA}}(N, \alpha)= & \exp \left(\frac{\psi^{2}-4 N \sigma^{2} \alpha z}{8\left(N \sigma^{2} \alpha\right)^{2}}\right) Q\left(\frac{\psi^{2}-2 N \sigma^{2} \alpha z}{2 N \sigma^{2} \alpha \psi}\right) \\
& +Q\left(\frac{z}{\psi}\right),
\end{aligned}
$$

where $\psi=\sigma^{2} \sqrt{N}, z=\lambda-N \sigma^{2}$ with $\lambda$ denoting the decision threshold, $\alpha=\sigma_{t}^{2} / \sigma_{d}^{2}$ denotes the training-to-information ratio (TIR) with $\sigma_{t}^{2}$ representing the average power in the training sequence, and $Q(\cdot)$ is the Q-function or complementary cumulative distribution function of the standard Gaussian distribution. On the other hand, the probability of detection (i.e., the probability of correctly decides the presence of the $\mathrm{PU})$ is given by

$$
P_{\mathrm{D}}(N, \alpha)=\int_{0}^{\infty} Q\left(\frac{\lambda-\xi}{\psi_{1}}\right) \frac{1}{2} e^{\frac{-1}{2}(\chi+\rho)} I_{0}(\sqrt{\rho \chi}) d \chi,
$$

where $I_{0}$ is the modified Bessel function of the first type and order zero, $\psi_{1}=\sqrt{2 N \theta^{2} \sigma_{t}^{2}\left(\theta^{2} \sigma_{d}^{2}+\sigma^{2}\right)+N\left(\theta^{2} \sigma_{d}^{2}+\sigma^{2}\right)^{2}}$, and $\xi=N \theta^{2}\left(\sigma_{d}^{2}+\sigma_{t}^{2}\right)+N \sigma^{2}+N \alpha\left(\theta^{2} \sigma_{d}^{2}+\sigma^{2}\right) \chi$ with $\chi$ representing a noncentral Chi-squared random variable with two degrees of freedom and with no-centrality parameter $\rho=$ $2 N \theta^{2} \sigma_{t}^{2} /\left(\theta^{2} \sigma_{d}^{2}+\sigma^{2}\right)$.

\section{ENERGY EFFICIENCY}

In the context of CR, the energy efficiency can be defined as the number of bits transmitted by the secondary user per unit of energy consumption. Thus, the energy efficiency function is given by [5]

$$
\eta_{\mathrm{EE}}(N, \alpha)=\frac{R(N, \alpha) \cdot N_{t}}{E(N, \alpha)},
$$

where $R(N, \alpha)$ represents the secondary user's average throughput and $E(N, \alpha)$ represents the average energy consumption, both depending on the number of samples used for spectrum sensing $\mathrm{N}$ and the TIR value $\alpha$, according to the considered scenario. In order to determine the SU's throughput and the energy consumption, the following four cases are considered:

1. The secondary user correctly decides the primary user's absence with probability $P\left\{\mathcal{H}_{0}\right\}\left(1-P_{\mathrm{FA}}(N, \alpha)\right)$, where $P\left\{\mathcal{H}_{0}\right\}$ is the prior probability of the PU being absent. In this case, the secondary user is allowed to transmit data. Then, the secondary user's throughput is given by

$$
R_{0}(N)=\log _{2}\left(1+\gamma_{s}\right)
$$

where $\gamma_{s}$ is the signal-to-noise ratio (SNR) for the secondary link (i.e. the secondary user transmission link), and the energy consumption can be calculated as

$$
E_{0}(N)=N\left(P_{s}+P_{c}\right)+\left(N_{t}-N\right)\left(P_{t}+P_{c}\right),
$$

where $P_{s}, P_{c}$, and $P_{t}$ are the sensing power, the circuit power, and the SU's transmission power, respectively.

2. The secondary user incorrectly decides the primary user's presence, i.e. a false alarm occurs, with probability $P\left\{\mathcal{H}_{0}\right\} P_{\mathrm{FA}}(N, \alpha)$. In this case, the secondary user does not transmit data and the energy consumption is given by

$$
E_{1}(N)=N\left(P_{s}+P_{c}\right) .
$$

3. The secondary user incorrectly decides the absence of the primary user, i.e. a missed detection occurs, with probability $P\left\{\mathcal{H}_{1}\right\}\left(1-P_{\mathrm{D}}(N, \alpha)\right)$, where $P\left\{\mathcal{H}_{1}\right\}=$ $1-P\left\{\mathcal{H}_{0}\right\}$ is the prior probability of the PU being present. Thus, the secondary user transmits data and the throughput is given by

$$
R_{1}(N, \alpha)=\log _{2}\left(1+\frac{\gamma_{s}}{1+\gamma_{p}}\right),
$$

where $\gamma_{p}$ is the SNR of the link between the primary user and the secondary user, and the energy consumption is given by (6).

4. The primary user is transmitting data and the secondary user correctly decides its presence with probability $P\left\{\mathcal{H}_{1}\right\} P_{\mathrm{D}}(N, \alpha)$. In this case, the secondary user is not allowed to transmit data and the energy consumption is given by (7).

Therefore, the secondary user's throughput is given by

$$
\begin{array}{r}
R(N, \alpha)=\left(\frac{N_{t}-N}{N_{t}}\right) P\left\{\mathcal{H}_{0}\right\}\left(1-P_{\mathrm{FA}}(N, \alpha)\right) R_{0} \\
\quad+\left(\frac{N_{t}-N}{N_{t}}\right) P\left\{\mathcal{H}_{1}\right\}\left(1-P_{\mathrm{D}}(N, \alpha)\right) R_{1}(N, \alpha),
\end{array}
$$

and the average energy consumption is

$$
\begin{aligned}
& E(N, \alpha)=N\left(P_{s}+P_{c}\right)+\left[P\left\{\mathcal{H}_{0}\right\}\left(1-P_{\mathrm{FA}}(N, \alpha)\right)\right. \\
& \left.\quad+P\left\{\mathcal{H}_{1}\right\}\left(1-P_{\mathrm{D}}(N, \alpha)\right)\right] \times\left[N_{t}-N\left(P_{t}+P_{c}\right)\right] .
\end{aligned}
$$

Note that in (9), the first term in the equation considerably contributes more to the throughput than the second term since when a sufficient protection to PU's transmissions is considered, the value of $P_{\mathrm{D}}$ is large. Additionally, the value of $P_{\mathrm{FA}}$ is expected to be small enough to exploit the greater number of spectrum opportunities. Thus, only the first term in the equation can be used as an approximation of the throughput [8].

\section{A. Cooperative Spectrum Sensing Scenario}

The detection performance in a CR network can be improve when two or more SUs collaborate between them in spectrum sensing. Improving the detection performance increases the throughput in the SU's network, which in consequence impacts on the energy efficiency. Thus, this section describes the energy efficiency function for cooperative spectrum sensing. 
Consider a CR scenario with a PU operating in a frequency band, K cooperative SUs and a fusion center (FC). Additionally, consider that each secondary user periodically senses the PU's frequency band and sends its local sensing decision to the FC by means of a control channel. Then, the FC combines the received results and makes a global decision about the presence or absence of the PU based on a OR-rule, i.e., the FC decides the presence of the $\mathrm{PU}$ is any of the $\mathrm{K}$ individual results is $\mathcal{H}_{1}$. Otherwise, the FC decides the absence of the PU. Thus, the overall probability of false alarm is given by [3]

$$
Q_{\mathrm{FA}}(N, \alpha, K)=1-\left(1-P_{\mathrm{FA}}(N, \alpha)\right)^{K},
$$

and the overall probability of detection is given by

$$
Q_{\mathrm{D}}(N, \alpha, K)=1-\left(1-P_{\mathrm{D}}(N, \alpha)\right)^{K},
$$

where $P_{\mathrm{FA}}(N, \alpha)$ and $P_{\mathrm{D}}(N, \alpha)$ are given by (2) and (3), respectively. Furthermore, consider the frame structure of cooperative spectrum sensing shown in Fig. 4, where $N_{r, k}$ is the reporting time of the $k$-th cooperative $\mathrm{SU}$, with $1 \leq$ $k \leq K$. Since the reporting time is assumed to be equal $\forall k$, it is denoted by $N_{r}$ to simplify notations. Thus, the average throughput of the $\mathrm{CR}$ network is given by

$$
\begin{aligned}
R(N, \alpha, K)= & \left(\frac{N_{t}-N-K N_{r}}{N_{t}}\right) . \\
& P\left\{\mathcal{H}_{0}\right\}\left[1-Q_{\mathrm{FA}}(N, \alpha, K)\right] R_{0} \\
& +\left(\frac{N_{t}-N-K N_{r}}{N_{t}}\right) . \\
& P\left\{\mathcal{H}_{1}\right\}\left[1-Q_{\mathrm{D}}(N, \alpha, K)\right] R_{1}(N, \alpha, K),
\end{aligned}
$$

and the energy consumption in this case is given by

$$
\begin{gathered}
E(N, \alpha, K)=K N P_{s}+K N_{r} P_{r}+K\left(N+N_{r}\right) P_{c}+ \\
{\left[P\left\{\mathcal{H}_{0}\right\}\left(1-Q_{F A}(N, \alpha, K)\right)+P\left\{\mathcal{H}_{1}\right\}\left(1-Q_{\mathrm{D}}(N, \alpha, K)\right)\right]} \\
\times\left[N_{t}-N-K N_{r}\left(P_{t}+P_{c}\right)\right] .
\end{gathered}
$$

Finally, for cooperative spectrum sensing, (13) and (14) are substituted in the energy efficiency function given by (4).

\section{Computer Simulations Results}

In this section, the energy-efficiency and its relationship with the spectrum-efficiency, in terms of the SU's achievable throughput, is analyzed through Monte Carlo simulations. The analyses are carried out using the traditional energy detection and the simplified SCAR-Phase sensing. For both, the noncooperative spectrum sensing and the cooperative spectrum sensing scenarios, it is considered that the PU employs ST, implemented as illustrated in Fig. 1, for channel estimation in its own network and the training sequence superimposed (i.e., added) to the data sequence is designed as in [9], which is given by $t[n]=\sigma_{t} e^{j \frac{\pi}{P}[n(n+u)]}$ for $n=0,1, \cdots P-1$ with $u=1$ for $P$ odd or $u=2$ for $P$ even, where $P$ denotes the period of the training sequence. The results are obtained assuming that $P=10, \alpha=\sigma_{t}^{2} / \sigma_{d}^{2}=0.2$, and $\sigma_{d}^{2}+\sigma_{t}^{2}=1$. Additionally, the data sequence is unknown by the SUs with each sample $d[n]$ assumed to have the following distribution:

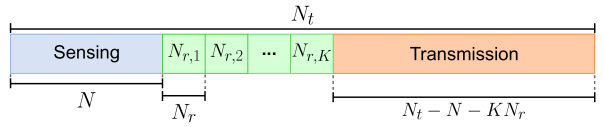

Fig. 4. Frame structure of cooperative spectrum sensing.

$d[n] \sim \mathcal{C N}\left(0, \sigma_{d}^{2}\right)$. Thus, it is considered that the received signal at each SU is distributed according to (1) with random channel gains, where the training sequence is known by the $\mathrm{SU}$ and $\tau$ is unknown. Hence, the value of $\tau$ in (1) is an integer obtaining from a uniform distribution with interval $[0, P-1]$ in such a way that it variates in each Monte Carlo simulation and then, it is estimated according to the synchronization method used in the simplified SCAR-Phase sensing described in [6]. The noise variance is given by $\sigma^{2}=\theta^{2}\left(\sigma_{d}^{2}+\sigma_{t}^{2}\right) / \gamma$ and it is chosen to satisfy a determined value of instantaneous SNR, denoted by $\gamma$. The CR scenario is considered as in [8], in which the link between the PU transmitter and the SU receiver is in the very-low SNR region, where the detection of the PU's signal can be very tough due to effects such as the hidden terminal problem, shadowing and fading. Therefore, it is considered that $\gamma_{p}=-12 \mathrm{~dB}$, unless otherwise stated. On the contrary, the SUs are considered to be operating under less severe conditions where communication is still possible. Thus, it is assumed that $\gamma_{s}=5 \mathrm{~dB}$. Moreover, the prior probability of the PU being absent is set to $P\left\{\mathcal{H}_{0}\right\}=0.7$ (thus, $P\left\{\mathcal{H}_{1}\right\}=0.3$ ) and the target probability of detection is set to $P_{\mathrm{D}}=\beta_{d}=0.9$. Thus, the threshold that satisfies $\beta_{d}$ is used to obtain the values of the probability of false alarm used in the results. Finally, it is considered that the transmission power is $P_{t}=1 \mathrm{~W}$, and the values for the sensing power and circuit power are $P_{s}=40 \mathrm{~mW}$, and $P_{c}=80 \mathrm{~mW}$, respectively.

\section{A. Single SU based Spectrum Sensing}

For a scenario with one PU and one SU, it is considered the periodic spectrum sensing scheme shown in Fig. 2 with a total frame duration of $N_{t}=20,000$ samples. As shown in [6], the simplified SCAR-Phase sensing attains a better detection performance than the energy detection for a small number of samples under a low SNR scenario. Hence, when using this enhanced method, the SU might achieve lower values of probability of false alarm (in comparison with the energy detector), which improves its throughput as can be deduced from (9). The latter can be seen in Fig. 5 where the SU's throughput is plotted as a function of the sensing samples $N$. It can be observed in this figure that for small number of samples (e.g., less than 2,000), the simplified SCAR-Phase sensing attains higher throughput. Inclusively, the maximum throughput is achieved with a lower number of samples, compared to the energy detection method. On the other hand, as shown in Fig. 6, the average energy consumption first increases as the number of sensing samples increases, given that the probability of false alarm decreases, and the energy consumed in data transmission contributes more in (10). However, there is a given number of sensing samples after which the energy 


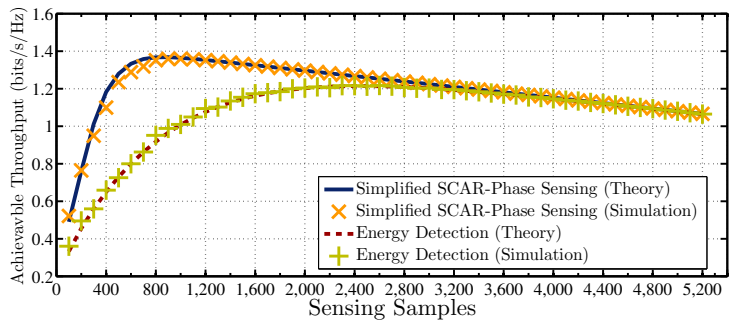

Fig. 5. Achievable SU's throughput as a function of sensing samples $N$.

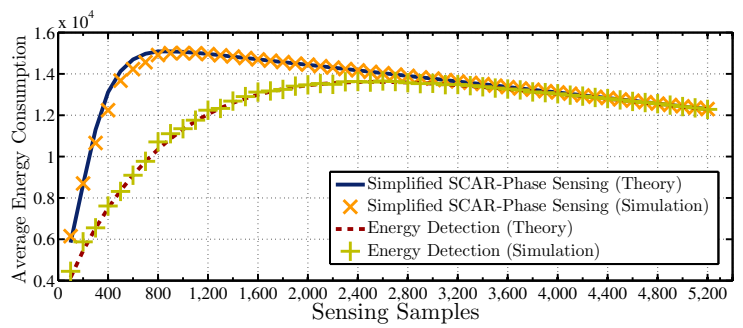

Fig. 6. Average energy consumption (in $\mathrm{W} \cdot$ samples) as a function of the number of sensing samples $N$.

consumption decreases since, although the probability of false alarm is still decreasing, the time used for data transmission is reduced. Note that although the simplified SCAR-Phase sensing has a greater energy consumption with small values of $N$ (approximately $N<3,000$ ) in comparison to the energy detection, it also has greater values of SU's throughput (as explained in Fig. 5). Hence the energy-efficiency might be improved when using the simplified SCAR-Phase sensing, as shown in Fig. 7, where it can be noticed that, for lower values of $N$ (approximately $N<2,200$ ), the simplified SCARPhase sensing has greater values of energy-efficiency than the energy detection. Inclusively, the maximum value of energyefficiency is attained with a smaller number of sensing samples ( $N \approx 600$ when using the simplified SCAR-Phase sensing) than that for the energy detection that has the maximum value of throughput in $N \approx 1,400$. Also note that the maximum value of energy-efficiency for both sensing methods (see Fig. 7) is achieved with a smaller number of sensing samples than that required to attain the maximum value of throughput (see Fig. 5). The latter can be easily seen in Fig. 8, where the number of required sensing samples to achieve the maximum energy-efficiency and the maximum SU's throughput for both sensing methods is plotted for different values of SNR. For both sensing methods, the number of required sensing samples to achieve the maximum energy-efficiency is lower than those required to achieve the maximum throughput (in the range of SNR shown). For example, under the scenario of $S N R=-15$ $\mathrm{dB}$, the maximum energy-efficiency is attained at $N=3,100$, while the maximum throughput is attained at $N=5,200$, both values for the energy detection. For the simplified SCARPhase sensing, the maximum energy-efficiency is reached at $N=1,250$, while the maximum throughput is reached at $N=1,500$. Thus, maximizing the energy-efficiency might

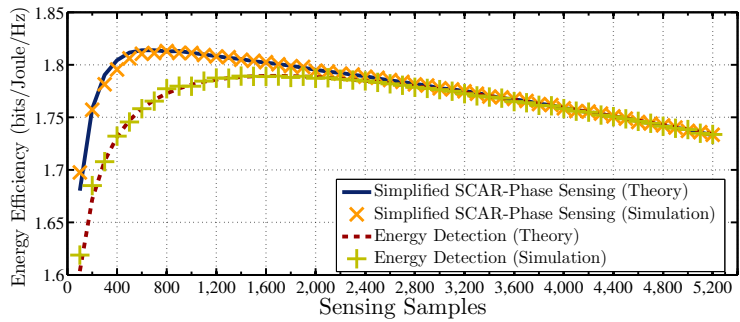

Fig. 7. Energy-efficiency as a function of the number of sensing samples, $N$.

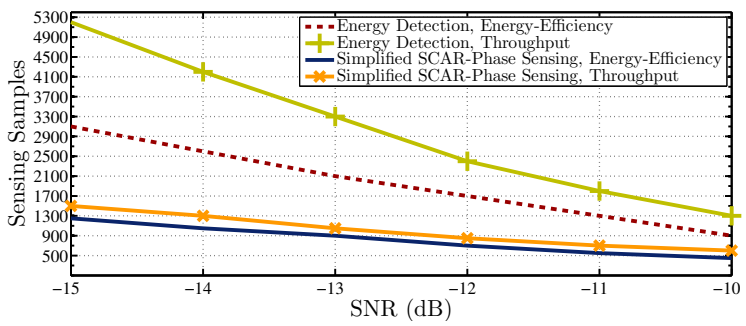

Fig. 8. Required sensing samples to achieve the maximum energy-efficiency and the maximum SU's throughput as a function of the SNR.

imply a reduction on the spectrum-efficiency (in terms of SU's throughput). The latter is observed in Fig. 9, where the maximum value of throughput (obtained through a linear search) for different levels of SNR is plotted and the attained throughput when the focus is obtaining the maximum energyefficiency, i.e., the achieved throughput with the number of samples that maximizes the energy-efficiency. What is important to note here is that, when using the simplified SCARPhase sensing, the obtained value of throughput is closer to the maximum throughput than the obtained by the energy detection. Thus, using the simplified SCAR-Phase sensing might improve the energy-efficiency without compromising by much the spectrum-efficiency.

\section{B. Cooperative Spectrum Sensing}

For the results in this section, $K$ SUs are considered and a fusion center with an OR decision rule, thus the overall detection performance is given by (11) and (12). The total frame duration is $N_{t}=20,000$ and the reporting time is assumed to be very short, thus $N_{r}=1$ sample and the power of reporting is $P_{r}=10 \mathrm{~mW}$. Other parameters are as previously specified. When multiple SUs cooperate in spectrum sensing, the overall detection performance is improved, as shown in Fig. 10, where the probability of false alarm decreases when more SUs cooperate in spectrum sensing. Thus, the throughput in the network is improved, as seen in Fig. 11. In this figure, the achievable throughput as a function of the sensing samples is plotted for $K=2$ and $K=6$ cooperative SUs. For both sensing methods, it can be observed the improvement when $K=6$ in comparison to $K=2$. However, the achievable throughput is greater when the simplified SCAR-Phase is used, for both $K=2$ and $K=6$, than that for the energy detection method. Thus, using the simplified SCAR-Phase sensing under 


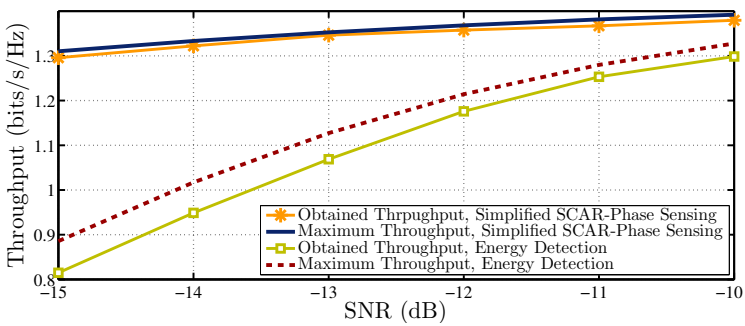

Fig. 9. Comparison between the maximum achieved SU's throughput and that obtained when maximizing the energy-efficiency.

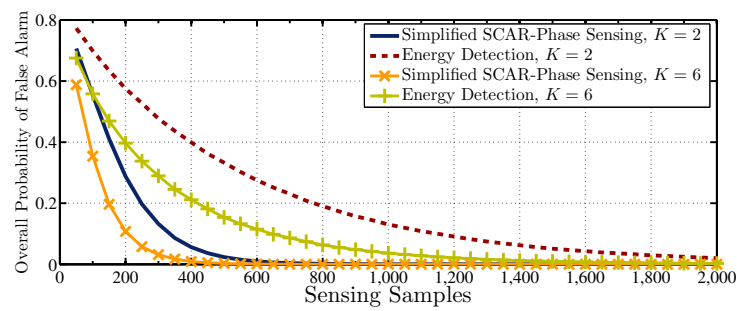

Fig. 10. Overall probability of false alarm as a function of the sensing samples. The results are for a target overall probability of detection $\beta_{d}=0.9$.

a cooperative spectrum sensing scenario might improve the energy-efficiency. This can be seen in Fig. 12, where the energy-efficiency as a function of the sensing samples for both sensing methods is displayed. It can be seen in this figure that the SCAR-Phase sensing obtains a greater energy-efficiency in its maximum point than the obtained by the energy detection, even with $K=2$ SUs. Note here that with more SUs, the maximum energy-efficiency value decreases since the energy consumed in spectrum sensing, as well as the energy consumed in reporting the local decisions, increases with the number of cooperative SUs. Thus, even the spectrum-efficiency is improved, the energy-efficiency might not when more SUs cooperate.

\section{CONCLUSIONS}

In this paper, the energy-efficiency of the simplified SCARPhase sensing and the energy detection spectrum sensing method was analyzed to show the advantages of exploiting the ST technique in this context. Moreover, the advantages in terms of the spectrum-efficiency when the goal is maximizing the energy-efficiency were shown. In this case, the results show that the impact in the spectrum efficiency is lower when using the SCAR-Phase sensing. Thus, the SCAR-Phase sensing might be used to improve the energy-efficiency with less reduction in the spectrum-efficiency. For the cooperative spectrum sensing scenario, it was shown that, for both sensing methods, the spectrum-efficiency is improved with more SUs. but the energy-efficiency is not improved, given the increment on the energy consumed during the sensing and reporting phases. However, it is important to note that the SCARPhase sensing showed the greater values of maximum energyefficiency, thus showing the advantages of exploiting the ST information in both, spectrum and energy-efficiency. More

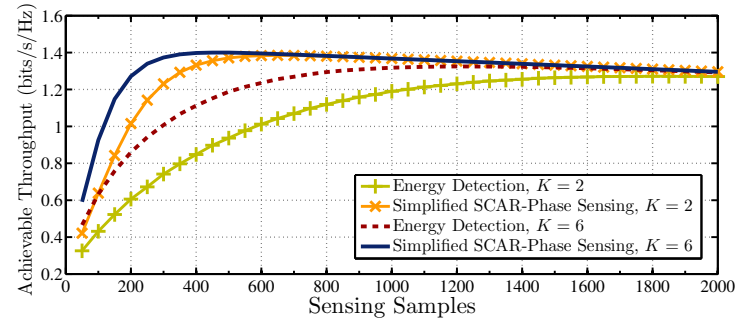

Fig. 11. Achievable throughput as a function of the sensing samples for cooperative spectrum sensing.

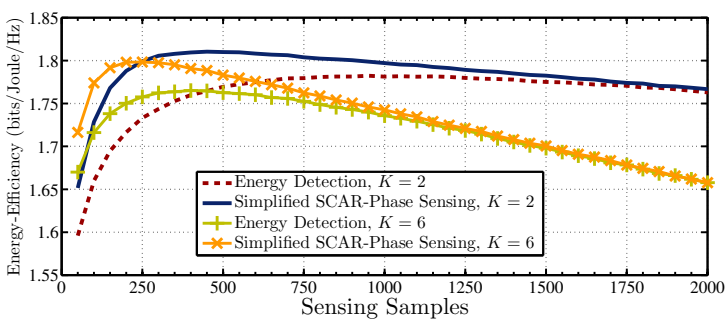

Fig. 12. Energy efficiency as a function of the number of sensing samples for different number of cooperative SUs.

studies about the tradeoff between the spectrum and the energy-efficiency are needed under a ST scenario.

\section{ACKNOWLEDGMENT}

This work has been partially supported by the Mexican National Council for Science and Technology (CONACYT).

\section{REFERENCES}

[1] K. M. S. Huq, S. A. Busari, J. Rodriguez, V. Frascolla, W. Bazzi and D. C. Sicker, "Terahertz-Enabled Wireless System for Beyond-5G UltraFast Networks: A Brief Survey," in IEEE Network, vol. 33, no. 4, pp. 89-95, July/August 2019.

[2] F. Awin, E. Abdel-Raheem and K. Tepe, "Blind Spectrum Sensing Approaches for Interweaved Cognitive Radio System: A Tutorial and Short Course," in IEEE Communications Surveys \& Tutorials, vol. 21, no. 1, pp. 238-259, Firstquarter 2019.

[3] S. Bae, J. So, H. Kim, (2017). "On Optimal Cooperative Sensing with Energy Detection in Cognitive Radio," Sensors, vol. 17, no. 9, pp. 2111, September 2017.

[4] K. Cichon, A. Kliks and H. Bogucka, "Energy-Efficient Cooperative Spectrum Sensing: A Survey," in IEEE Communications Surveys \& Tutorials, vol. 18, no. 3, pp. 1861-1886, thirdquarter 2016.

[5] H. Hu, H. Zhang and Y. Liang, "On the Spectrum- and EnergyEfficiency Tradeoff in Cognitive Radio Networks," in IEEE Transactions on Communications, vol. 64, no. 2, pp. 490-501, Feb. 2016.

[6] L. Lopez-Lopez, M. Cardenas-Juarez, E. Stevens-Navarro, U. PinedaRico, A. Arce, A. G. Orozco-Lugo, "Superimposed Training Combined Approach for a Reduced Phase of Spectrum Sensing in Cognitive Radio," Sensors, vol. 19, no. 11, pp. 2425, May 2019.

[7] E. Alameda-Hernandez, D. C. McLernon, A. G. Orozco-Lugo, M. M. Lara and M. Ghogho, "Frame/Training Sequence Synchronization and DC-Offset Removal for (Data-Dependent) Superimposed Training Based Channel Estimation," in IEEE Transactions on Signal Processing, vol. 55, no. 6, pp. 2557-2569, June 2007.

[8] Y. Liang, Y. Zeng, E. C. Y. Peh and A. T. Hoang, "Sensing-Throughput Tradeoff for Cognitive Radio Networks," in IEEE Transactions on Wireless Communications, vol. 7, no. 4, pp. 1326-1337, April 2008.

[9] A. G. Orozco-Lugo, M. M. Lara and D. C. McLernon, "Channel estimation using implicit training," in IEEE Transactions on Signal Processing, vol. 52, no. 1, pp. 240-254, Jan. 2004. 